\title{
A statistical study of shear motion of EUV footpoints in two-ribbon flares
}

\author{
Y. N. Su ${ }^{1,2,3}$, L. Golub ${ }^{1}$, A. A. Van Ballegooijen ${ }^{1}$, and G. L. Huang ${ }^{2}$ \\ ${ }^{1}$ Harvard-Smithsonian Center for Astrophysics, Cambridge, MA 02138, USA \\ email: ynsu@head.cfa.harvard.edu \\ ${ }^{2}$ Purple Mountain Observatory, Nanjing, 210008, China \\ ${ }^{3}$ Graduate University of Chinese Academy of Sciences, China
}

\begin{abstract}
We present a statistical investigation of shear motion of the EUV footpoints in tworibbon solar flares, using the high spatial resolution data obtained in 1998-2005 by the Transition Region and Coronal Explorer (TRACE). In this study we have selected 50 well-observed X- and M- class two-ribbon flares. Our preliminary results are: a) 86\% (43 out of 50) of these flares show shear change of footpoints and ribbon separation; b) there is no obvious ribbon separation in those 7 flares which have no shear change of the conjugate footpoints; c) in most events, the cessation of shear change is 0-2 minutes earlier than the end of the impulsive phase.
\end{abstract}

\section{Introduction}

It is well known that during a two-ribbon flare the two footpoint ribbons, residing in opposite magnetic polarities, expand outward and away from each other. However, the shear motion of the footpoints along the direction parallel to the magnetic inversion line (MIL) has only been examined by several authors in some individual two-ribbon flares (for a review, see $\mathrm{Su}$ et al. 2006). This motion, which often appears as strong-to-weak shear change of the footpoints during a flare, is interpreted by a magnetic field model with highly sheared inner and less sheared outer magnetic field configuration during the preflare phase, and the cessation of shear change during the impulsive phase is interpreted as a splitting of the envelope of the highly sheared core field ( $\mathrm{Su}$ et al. 2006).

\section{Observations and Discussion}

We have identified 50 well-observed two-ribbon solar flares listed in the TRACE Flare Catalog from 1998 to 2005 according to the following criteria: (1) we only consider flares in which two long and roughly parallel ribbons are seen during the flare; (2) most parts of the two ribbons are visible within the field of view (FOV) of TRACE; (3) TRACE has obtained several good images during the rise and impulsive phase, from which we can see the two ribbons and their evolution clearly; (4) flares near the limb for which the two ribbons and their evolution cannot be seen are not considered.

The shear change of the footpoints and ribbon separation have been found in $86 \%$ (43 out of 50) of the flares we have selected. The initial and final shear angles of 25 flares have been measured. Figures 1a-1d shows an example of how to measure these angles: firstly, look through all the TRACE images to select two images with the initial (at the early beginning of the flare) and final (at the time when the shear change stops) conjugate footpoints (identified by the post flare loop (Figures 1a and 1b)); secondly, measure the initial and final angles between the line connecting the two conjugate footpoints and the magnetic inversion line (MIL, SoHO/MDI), i.e., two white lines in Figures 1c 

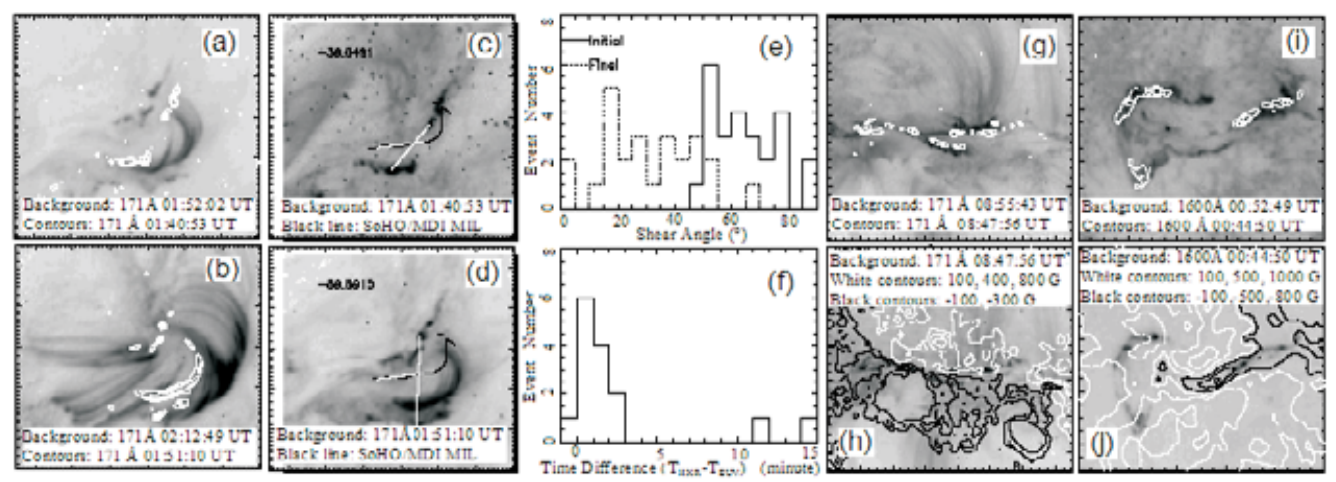

Figure 1. $(a),(b),(c),(d)$ TRACE images of event 2002 July $31 ;(e),(f)$ histograms of events with shear change; $(g),(h)$ TRACE and SoHO/MDI images of event 2001 May 05; $(i),(j)$ similar to $(g),(h)$ but for event 2001 November 10.

and 1d). Note that the shear angle is complementary to the angle we have measured. The histogram of the initial and final shear angles (for 25 flares with measured shear angles) in terms of the event number (Figure 1e) shows that the initial and final angles in most events are in the range $50^{\circ}-80^{\circ}$ and $15^{\circ}-55^{\circ}$, respectively.

In order to answer the question: could the change from the impulsive to gradual phase be related to the magnetic shear change, we find 15 events within the 25 events with measured shear angle and having corresponding HXR (Yohkoh/HXT or RHESSI) observations (which provide the information about when the impulsive phase stops). The histogram of the time difference between the end of the HXR impulsive phase and the cessation of the shear change (Figure 1f) indicates that in most events, the cessation of shear change is 0-2 minutes earlier than the time when the impulsive phase stops.

We have not found obvious ribbon separation in those 7 flares which show no obvious shear change of the footpoints. We divide these flares into three types. A filament can be seen in the $\mathrm{H} \alpha$ images (Big Bear Solar Observatory) corresponding to Type I flares (2 events) which have small ribbon separation (i.e., Figure 1g). These flares have singlebipole magnetic field configuration (Figure 1h); may also be caused by the release of energy stored in the highly sheared magnetic field region. Filaments are not seen in Type II flares (3 events) and no ribbon separation can be seen either (Figure 1i). Similar to compact flares, these flares could be explained by the emerging (or evolving) flux model (Heyvaerts et al. 1977), because they have complicated magnetic field configuration (multi-polarity, Figure 1j). Similar to type II flares, the type III flares (2 events) have single-bipole magnetic field configuration. It is not clear what the mechanism is, but it seems that these flares are very similar to the single-loop (compact) flares.

Acknowledgments: The authors wish to thank the team of TRACE, SoHO/MDI, NJIT/BBSO, Yohkoh/HXT, and RHESSI for providing the valuable data. The TRACE analysis was supported at Smithsonian Astrophysics Observatory (SAO) by a contract from Lockheed Martin. Y.N. Su was also supported by SAO Predoctoral Fellowship and the NSFC projects No. 10333030 and 10273025.

\section{References}

Heyvaerts, J., Priest, E.R. and Rust, D.M. 1977, ApJ. 216, 123.

Su, Y.N., Golub, L., Van Ballegooijen, A.A., \& Gros, M. 2006, Solar Phys., accepted. 